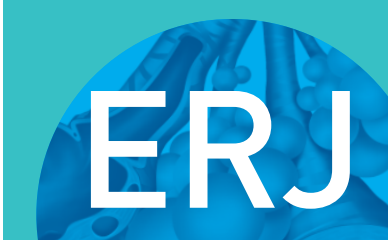

open research
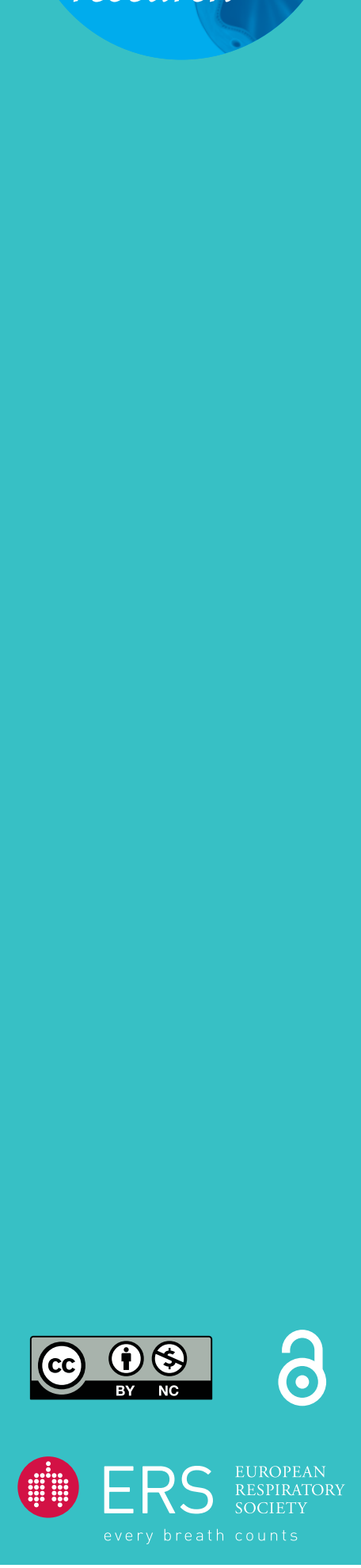

\section{Factors predicting progression of exercise training loads in people with interstitial lung disease}

\section{To the Editor:}

People with interstitial lung disease (ILD) experience dyspnoea on exertion, poor exercise capacity and reduced health-related quality of life. Whilst new pharmaceutical treatments slow disease progression in some patients, most care options remain supportive $[1,2]$. Pulmonary rehabilitation (PR) is recommended for people with ILD, however not all participants have a positive response [3-5]. A recent randomised controlled trial found that the benefits of exercise training were greatest in individuals who were able to progress their exercise training loads according to the study protocol [4]. The aim of this analysis was to identify predictors of the ability to adhere to the exercise progression protocol in people with ILD.

This study is a secondary analysis of a larger randomised controlled trial, in which people with ILD were recruited from three hospitals in Melbourne, Australia (ACTRN12611000416998). Detailed methods are described elsewhere [4]. Participants who were randomly allocated to exercise training undertook PR twice a week for 8 weeks. The supervised outpatient exercise training programme consisting of 30 min of aerobic exercise, cycling and walking, plus upper and lower limb resistance training. Randomisation was stratified according to four ILD sub-groups: i) idiopathic pulmonary fibrosis (IPF), ii) dust-related ILD, iii) connective tissue disease-related ILD, and iv) other ILD. Assessments were completed at baseline, at the end of pulmonary rehabilitation and at 6-month follow-up. Outcome measures included 6-minute walk test, Chronic Respiratory Disease Questionnaire, St George Respiratory Questionnaire IPF specific version, University of California San Diego Shortness of Breath Questionnaire, modified Medical Research Council dyspnoea score, and Hospital Anxiety and Depression Scale. Respiratory function tests and transthoracic echocardiography were also performed.

Initial walking training intensity was set at $80 \%$ of the peak walking speed during baseline 6-minute walk test and increased each week by $0.25-0.5 \mathrm{~km} \cdot \mathrm{h}^{-1}$, depending on initial speed, if the target rate of perceived exertion during training was Borg scale range of 3-4 [4]. When walking speed reached $4-5 \mathrm{~km} \cdot \mathrm{h}^{-1}$, speed was reduced by $0.2-0.4 \mathrm{~km} \cdot \mathrm{h}^{-1}$ and a $1-2 \%$ incline was added weekly. Successful exercise progression was evaluated based on the walking training component of the programme as we had previously shown this to be an important determinant of programme outcomes [4], and was defined as completion of the walking training protocol on at least five out of seven possible weeks (weeks 2-8 of the rehabilitation programme).

Statistical analysis was performed using IBM SPSS Statistics 24 (SPSS, Chicago, IL, USA). Outcomes were categorised into those who did (progressors) and those who did not (non-progressors) adhere to the exercise training protocol. Normally distributed data were analysed with the Student t-test and Pearson Chi-squared test, whereas non-normally distributed data were analysed with the Mann-Whitney U-test. Logistic regression was used to identify independent predictors of exercise progression. As there were no established associations between patient features and exercise progression, we used univariate analysis to identify potential predictors. Variables included in univariate analysis were age, diagnosis, respiratory function, pulmonary artery systolic pressure, exertional desaturation, dyspnoea and programme attendance.

$@$ ERSpublications

In ILD, adherence to the training sessions in pulmonary rehabilitation predicts progression of exercise training loads; declining lung function is an independent predictor of failure to progress training loads http://bit.ly/2Z4x9Nw

Cite this article as: Nakazawa A, Dowman LM, Cox NS, et al. Factors predicting progression of exercise training loads in people with interstitial lung disease. ERJ Open Res 2019; 5: 00245-2018 [https://doi.org/10.1183/23120541.00245-2018].

Copyright $\odot$ ERS 2019. This article is open access and distributed under the terms of the Creative Commons Attribution NonCommercial Licence 4.0. 
All variables that had a significant association with progression on univariate analysis were included in the model and no variables were removed. A p-value $<0.05$ was considered significant.

74 participants were randomised to exercise training. However, five people attended no session after their enrolment, leaving 69 participants in the current analysis (male $n=41$, mean \pm SD age $68 \pm 10$ years and forced vital capacity $74 \pm 19 \%$ predicted; IPF $n=31$, dust related ILD $n=9$, connective tissue disease-related ILD $n=10$, other ILD $n=19$; progressors $n=32)$. Exercise progressors $(n=32)$ had less dyspnoea with lower baseline modified Medical Research Council score (1.4 \pm 0.7 versus $1.8 \pm 0.9 ; \mathrm{p}=0.04$ ). Individuals with a diagnosis other than IPF accounted for a greater proportion of progressors than non-progressors (72 versus $41 \% ; \mathrm{p}=0.009)$. Progressors attended more exercise sessions than non-progressors $(14 \pm 1$ versus $8 \pm 5$ sessions; $\mathrm{p}<0.001)$ and demonstrated a slight improvement in lung carbon monoxide transfer factor $\left(T_{\mathrm{LCO}}\right)$ over 6-month follow-up compared with a small reduction in non-progressors $(0.76 \pm 2.1$ versus $\left.-1.00 \pm 2.4 \mathrm{~mL} \cdot \mathrm{min}^{-1} \cdot \mathrm{mmHg}^{-1} ; \mathrm{p}=0.005\right)$. Decline in FVC was not associated with progression. There was no difference between progressors and non-progressors in baseline pulmonary artery systolic pressure (32.1 \pm 14.4 versus $33.1 \pm 11.9 \mathrm{mmHg} ; \mathrm{p}=0.817)$. Logistic regression analysis showed that attending a greater number of PR sessions and less reduction in $T_{\mathrm{LCO}}$ over 6-month follow-up were independent predictors of adherence to the exercise progression protocol (table 1). The modified Medical Research Council score at baseline and diagnosis of IPF (versus other diagnoses) were not independent predictors of progression.

This is the first paper to describe factors predicting the ability to progress exercise training loads in people with ILD. We found that baseline clinical and demographic features did not predict the likelihood of exercise progression. Attendance at a greater number of training sessions is a logical predictive factor given that progression in exercise training load can only occur at PR sessions. Importantly, those with a more rapid decline in $T_{\mathrm{LCO}}$ were unlikely to progress their programme, which has not been confirmed in the setting of ILD so far. The same relationship was not seen for FVC. It is possible that worsening pulmonary vascular disease is a more important determinant of tolerance of exercise training loads than lung capacity, although we note that pulmonary artery systolic pressure was not a significant predictor.

At the time of referral to PR it may not be clear whether patients are experiencing worsening respiratory function. The clinical course of ILD varies, such that some patients may experience periods of stability whilst others may experience steady or intermittent periods of decline [6]. Many efforts are underway to identify a biomarker for those with rapid decline [7], but this is not yet available. Our data suggest that for clinicians conducting PR, the inability to progress exercise intensity over one or more weeks should be a warning sign for poor $\mathrm{PR}$ outcome, irrespective of whether a decline in lung function has been demonstrated. Similar to most other PR trials in ILD [8] this study used continuous exercise of moderate intensity, which has been adapted from training protocols used successfully in COPD [3, 9]. Our results suggest this may not be optimal for patients with ILD and declining lung function, who frequently demonstrate severe symptoms and marked oxygen desaturation. Investigation of other training protocols for ILD is warranted, such as interval training, which involves periods of relatively high intensity exercise interspersed with periods of lower intensity exercise, with the potential to minimise symptoms and oxygen desaturation [3]. It is also worthy of note that better response to PR is seen with referral early in the disease course [10], providing more opportunity to achieve sustained benefits before significant decline in lung function has occurred.

In conclusion, attending a greater number of sessions and less decline in respiratory function over 6 months were predictors of progression of exercise training loads during PR. Future research should

\begin{tabular}{|c|c|c|c|c|c|}
\hline & B & SE & p-value & Odds ratio & $95 \% \mathrm{Cl}$ \\
\hline Constant & -7.749 & 2.813 & 0.006 & & \\
\hline PR sessions attended & 0.676 & 0.212 & $0.001 *$ & 1.967 & $1.299-2.978$ \\
\hline Change in $T_{\text {LCo }} 6$ months after PR & 0.484 & 0.227 & $0.033^{*}$ & 1.623 & $1.041-2.531$ \\
\hline Baseline mMRC & -0.644 & 0.555 & 0.246 & 0.525 & $0.177-1.557$ \\
\hline $\begin{array}{l}\text { Diagnosis of IPF } \\
R^{2}\end{array}$ & 0.397 & 0.809 & 0.241 & 1.488 & $0.305-7.266$ \\
\hline
\end{tabular}

PR: pulmonary rehabilitation; $T_{\text {LCo: }}$ lung carbon monoxide transfer factor; mMRC: modified Medical Research Council score; IPF: idiopathic pulmonary fibrosis; $B$ : unstandardised coefficient; SE: standard error; $R^{2}$ : proportion of variance in difference between progression and non-progression in exercise training explained by the model. *: $p<0.05$. 
investigate strategies to optimise exercise training for individuals with ILD, especially those with worsening disease within the safe limitations.

Atsuhito Nakazawa (1) $^{1}$, Leona M. Dowman ${ }^{1,2,3,4}$, Narelle S. Cox ${ }^{1,4}$, Christine F. McDonald ${ }^{3,4,5}$, Catherine J. Hill ${ }^{2,4}$, Annemarie L. Lee ${ }^{4,6}$ and Anne E. Holland $\oplus^{1,4,7}$

${ }^{1}$ Discipline of Physiotherapy, La Trobe University, Alfred Centre, Melbourne, Australia. ${ }^{2}$ Dept of Physiotherapy, Austin Health, Heidelberg, Australia. ${ }^{3}$ Dept of Respiratory and Sleep Medicine, Austin Health, Heidelberg, Australia. ${ }^{4}$ Institute for Breathing and Sleep, Heidelberg, Australia. ${ }^{5}$ Dept of Medicine, University of Melbourne, Parkville, Australia. ${ }^{6}$ Dept of Physiotherapy, Monash University, Frankston, Australia. ${ }^{7}$ Dept of Physiotherapy, Alfred Health, Melbourne, Australia

Correspondence: Atsuhito Nakazawa, La Trobe University and Alfred Health, Level 4, The Alfred Centre, 99 Commercial Road, Melbourne, VIC 3004, Australia. E-mail: gda_kawasaki@yahoo.co.jp

Received: 12 Dec 2018 | Accepted after revision: 03 Aug 2019

This trial is registered at www.anzctr.org.au with identifier number ACTRN12611000416998. De-identified participant data are available from the authors on reasonable request, with oversight of the relevant human research ethics committee.

Support statement: No specific grant from funding agencies in the public, commercial or not-for-profit sectors was received for this research. L.M. Dowman received a National Health and Medical Research Council postgraduate scholarship (grant ID: GNT1017802); and the randomised controlled trial received grant funding from the Pulmonary Fibrosis Foundation/American Thoracic Society Foundation, Institute for Breathing and Sleep and Eirene Lucas Foundation. These funding agencies had no role in the management of this study. Other authors have no disclosures of funding. Funding information for this article has been deposited with the Crossref Funder Registry.

Conflict of interest: A. Nakazawa has nothing to disclose. L.M. Dowman has nothing to disclose. N.S. Cox has nothing to disclose. C.F. McDonald reports speaker fees from GSK, advisory board fees from Pfizer and Novartis, and speaker fees paid to her hospital from Menarini, outside the submitted work. C.J. Hill has nothing to disclose. A. Lee has nothing to disclose. A.E. holland has nothing to disclose.

\section{References}

1 Raghu G, Rochwerg B, Zhang Y, et al. An official ATS/ERS/JRS/ALAT clinical practice guideline: treatment of idiopathic pulmonary fibrosis. An update of the 2011 clinical practice guideline. Am J Respir Crit Care Med 2015; 192: e3-19.

2 Canestaro WJ, Forrester SH, Raghu G, et al. Drug treatment of idiopathic pulmonary fibrosis: systematic review and network meta-analysis. Chest 2016; 149: 756-766.

3 Spruit MA, Singh SJ, Garvey C, et al. An official American Thoracic Society/European Respiratory Society statement: key concepts and advances in pulmonary rehabilitation. Am J Respir Crit Care Med 2013; 188: e13-e64.

4 Dowman LM, McDonald CF, Hill CJ, et al. The evidence of benefits of exercise training in interstitial lung disease: a randomised controlled trial. Thorax 2017; 72: 610-619.

5 Nakazawa A, Cox NS, Holland AE. Current best practice in rehabilitation in interstitial lung disease. Ther Adv Respir Dis 2017; 11: 115-128.

6 Ley B, Collard HR, King TE, Jr. Clinical course and prediction of survival in idiopathic pulmonary fibrosis. Am J Respir Crit Care Med 2011; 183: 431-440.

7 Ley B, Brown KK, Collard HR. Molecular biomarkers in idiopathic pulmonary fibrosis. Am J Physiol Lung Cell Mol Physiol 2014; 307: L681-L691.

8 Dowman L, Hill CJ, Holland AE. Pulmonary rehabilitation for interstitial lung disease. Cochrane Database Syst Rev 2014; 10: CD006322.

9 American Collage of Sports Medicine. ACSM's guidelines for exercise testing and prescription. 9th Edn. Philadelphia, Wolters Kluwer Health/Lippincott Williams \& Wilkins, 2014.

10 Holland AE, Hill CJ, Glaspole I, et al. Predictors of benefit following pulmonary rehabilitation for interstitial lung disease. Respir Med 2012; 106: 429-435. 